\title{
Silicon Strain Gauge Load Cell for Weighting Disdrometer
}

\author{
Seon-Gil Lee ${ }^{1,3}$, Young-Soon Moon', Won-Ho Son², Young-Ho Sohn4, and Sie-Young Choi ${ }^{2,+}$
}

\begin{abstract}
In this paper, the usability of a compact silicon strain gauge load cell in a weighting disdrometer for measuring the impact load of a falling raindrop is introduced for application in a multi-meteorological sensor. The silicon strain gauge load cell is based on the piezoresistive effect, which has a high linearity output from the momentum of the raindrop and the simplicity of signal processing. The weighting disdrometer shows a high sensitivity of $7.8 \mathrm{mV} / \mathrm{g}$ in static load measurement when the diaphragm thickness of the load cell is $250 \mu \mathrm{m}$.
\end{abstract}

Keywords : Silicon strain gauge, Center boss diaphragm, Load cell, Disdrometer

\section{INTRODUCTION}

A disdrometer is a meteorological instrument used to measure the size of a raindrop and accumulated precipitation over a set period of time. In other words, it is a kind of rain gauge or precipitation sensor, which can calculate rainfall as well as rain intensity and rain depth in real time by measuring the size of each raindrop.

Commercially available disdrometers are classified by the principle of operation. Most conventional disdrometers are an acoustic detection type based on the piezoelectric effect. The piezoelectric transducer converts the acoustic signal into a voltage pulse, which is dependent on drop size, drop shape and terminal velocity from individual drop impacts [1]. The acoustic disdrometer is limited as to resolution, measurement range, and complicated signal processing. A new type of weighting disdrometer including a load cell of high sensitivity based on the piezoresistive effect is introduced in this paper. It converts the impact load of a raindrop into a proportional electrical signal via

${ }^{1}$ Dept. of Sensor and Display Eng., Kyungpook National University ${ }^{2}$ School of Electronics Eng., Kyungpook National University 1370 Sangyik-dong, Buk-gu, Daegu 702-701, Korea 'Daeyang Instrument Co., LTD.

503 Sinpyeong-dong, Saha-gu, Busan 604-030, Korea

${ }^{4}$ Dept. Of Computer Eng., Yeungnam University

280 Daehak-Ro, Gyeongsan-si, Gyeongbuk-do 712-749, Korea

${ }^{+}$Corresponding author: sychoi@ee.knu.ac.kr

(Received : Sep. 6, 2013, Revised : Sep. 24, 2013, Accepted : Sep. 24, 2013)

This is an Open Access article distributed under the terms of the Creative Commons Attribution Non-Commercial License(http://creativecommons.org/licenses/bync/3.0)which permits unrestricted non-commercial use, distribution, and reproduction in any medium, provided the original work is properly cited. elastic deformation of the silicon strain gauges.

Load cells are force sensors that are widely used throughout the industry as weighting equipment [2]. However, the sensitivity of a traditional load cell is limited by the metal foil strain gauge that is the common sensing element with a low gauge factor. In this weighting disdrometer, the silicon strain gauge was utilized as the sensing element of the load cell to perform at high sensitivity because the gauge factor of the silicon strain gauge is 20 to 100 times greater than that of a metal foil strain gauge [3]. In addition, an SUS630 metal diaphragm was used for the spring element of the load cell so it can withstand an impact load like unexpected hail. The spring element as the center boss diaphragm design performs with high linearity of output and precise low-load measurement corresponding to the load [4].

\section{DESIGN AND FABRICATION}

\subsection{Silicon strain gauge load cell}

The applied load significantly affects the strain on the surface of the diaphragm. When the load causes elastic deformation in the range of tensile strength, the amount of strain depends on the applied load, size of the diaphragm and the elasticity of the material.

The spring element of the load cell has been designed with a center bossed diaphragm used to reduce the nonlinearity in the pressure sensor or load cell. The spring element is a diaphragm of $8 \mathrm{~mm}$ diameter including a 
center boss of $2 \mathrm{~mm}$ diameter made of stainless steel grade 630 (SUS630 or AISI630) by computerized numerical control $(\mathrm{CNC})$ at a $\pm 10 \mu \mathrm{m}$ tolerance range. After surface grinding and lapping, thicknesses for the diaphragm were $150,200,250,300$ and $350 \mu \mathrm{m}$. Figure 1 shows a schematic view of the center boss diaphragm. The elastic modulus of SUS630 material is $196 \mathrm{GPa}$ and Poisson's ratio is 0.272 [5].

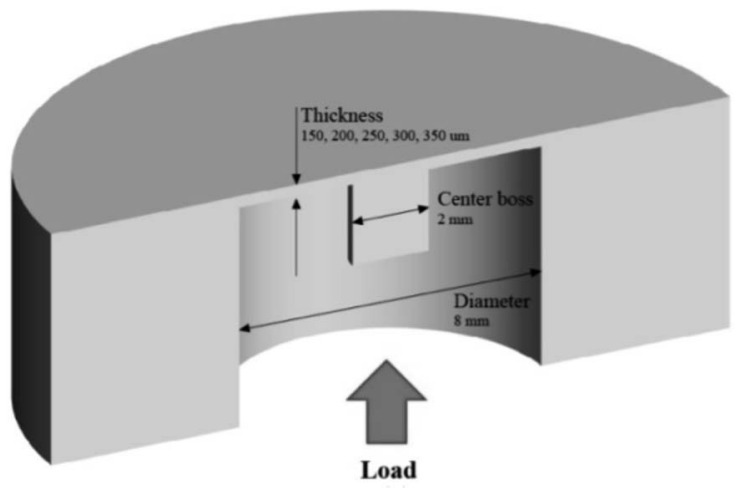

Fig. 1. Schematic view of the center boss diaphragm.

Sensitivity is an important consideration in the selection of the sensing element and is influenced by the gauge factor. Silicon strain gauges of high sensitivity were used for the sensing element of the load cell to measure a raindrop of less than $0.1 \mathrm{~g}$. The silicon strain gauges, fabricated by using a micro electro mechanical system (MEMS) process, were designed as linear patterns, as shown in Fig. 2. The main reason to select a linear design is that it is suitable for mass production and easy to align when installed on the surface of the spring element [6]. The piezoresistors were integrated at the four points of the single linear designed silicon strain gauge; tensile strain R1 and R3 are located at the edge of the center boss at a distance of $1.15 \mathrm{~mm}$ from the center of the diaphragm, and compressive strain R2 and R4 are located at the rim of the diaphragm at a distance of $3.8 \mathrm{~mm}$ from the center.

The p-type piezoresistors were fabricated by ion implantation of boron in the $<110>$ direction on the (100) plane of the n-type wafer in order to obtain the maximum piezoresistive effect [7]. The dose of the injected boron is $2.5 \times 10^{15} \mathrm{~cm}^{-2}$, and the acceleration energy is $70 \mathrm{keV}$. The average sheet resistance of the piezoresistors is $80.7 \Omega / \mathrm{cm}^{2}$ after the drive-in process at $1000^{\circ} \mathrm{C}$ during 60 minutes in a wet atmosphere. After the contact windows were open and $\mathrm{NiCr} / \mathrm{Au}$ deposited by the thermal evaporator, the electrodes were defined. The sensitivity of the strain gauges is represented by the gauge factor as determined by doping concentration in the silicon. To effectively detect the deformation of the diaphragm, the silicon strain gauge was machined by chemical-mechanical planarization (CMP) to a thickness of $100 \mu \mathrm{m}$.

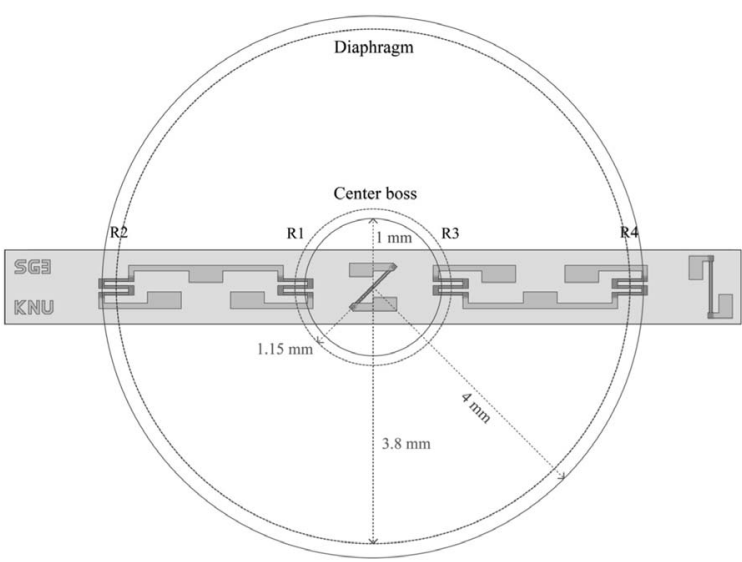

(a)

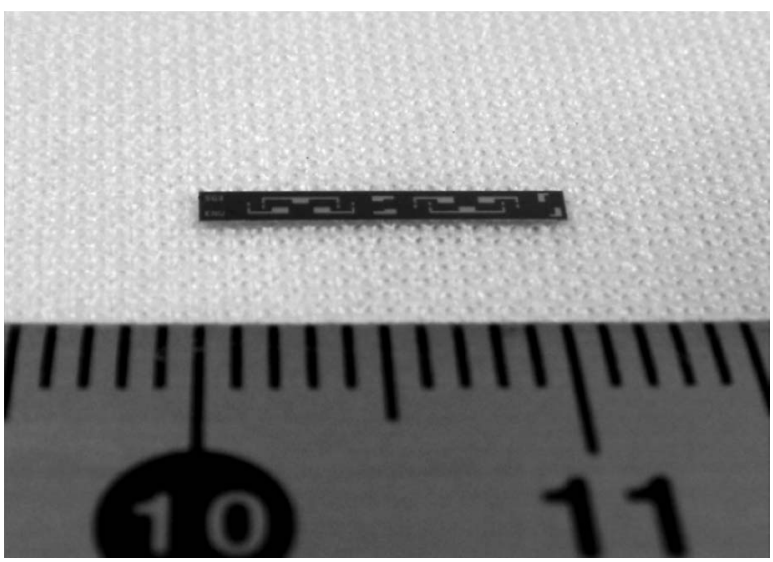

(b)

Fig. 2. The silicon strain gauge; (a) Schematic of the silicon strain gauge and (b) photo of the silicon strain gauge.

The silicon strain gauge was bonded to the surface of the diaphragm using M-bond 200. The electrodes in the PCB board and the piezoresistors in the strain gauge were connected by a wire bonder in order to form a wheatstone bridge, and the surface was coated with silicone rubber to protect the wire.

In a pressure sensor, if the strains are averaged over the region of the piezoresistors and if the gauge factor of the strain gauge is 2.0, the ratio of output voltage to supply voltage $\left(\Delta E / V_{i n}\right)$ can be approximated with Eq. (1) [8], where $\Delta E[\mathrm{mV}]$ is the change in load cell output, $V_{\text {in }}[V]$ is 
the applied voltage of the load cell, $\mathrm{k}_{0}$ is the pressure constant of the diaphragm, $P$ is the force to the unit area, $r$ is radius of the diaphragm, $V$ is Poisson's ratio of the material, $h_{d}$ is the thickness of the diaphragm and $E$ is elastic modulus of the material.

$$
\frac{\Delta E}{V_{i n}}=k_{0} \frac{\operatorname{Pr}^{2}\left(1-v^{2}\right)}{h_{d}^{2} E}
$$

Eq. (2), to approximate the output voltage of the load cell, can be derived by considering the mass of the object and the gauge factor of the strain gauge from Eq. (1). Simplify the Eq. (2) to obtain Eq. (3),

$$
\begin{aligned}
& \Delta E=k_{x} \frac{r^{2}\left(1-v^{2}\right)}{h_{d}^{2} E}\left(m_{0} A_{D}\right) \frac{h_{d}}{h_{d}+h_{s}} V_{i n} \frac{G F}{2} G_{v} \\
& \Delta E=K \frac{r^{2}\left(1-v^{2}\right)}{h_{d}\left(h_{d}+h_{s}\right) E} m_{0} A_{D} V_{i n} G F G_{v}
\end{aligned}
$$

where $K\left(k_{x} / 2\right)$ is the mass constant of the load cell, $\mathrm{m} 0$ is the mass, $A_{D}$ is the area of the diaphragm, hs is the thickness of the silicon strain gauge, GF is the gauge factor of the silicon strain gauge and $G_{v}$ is the amplification ratio of the amplifier circuit.

\subsection{Weighting disdrometer}

In the structure of the weighting disdrometer to measure the impact load of a falling water drop, the rain cap and the center boss of the load cell are connected by the impact bar, as shown in Fig. 3. The weighting disdrometer unit is made by aluminum alloy. The sampling area corresponding to the catchment space of the rain cap is $1809.6 \mathrm{~mm}^{2}$, and the weight of the rain cap, including the impact bar, is $9 \mathrm{~g}$.

The differential amplifier of the weighting disdrometer amplifies the output signal of the load cell caused by the change of loads by 100 times (R5/R6). The circuit diagram of differential amplifier can be seen in Fig. 4. The characteristics of the water droplets used in this experiment are described in Table 1. Water droplets of different mass were created by four kinds of syringe needle, and the average mass of the water drops was measured from weighing 100 drops. Water drops of weight ranging from 0.011 to 0.083 were generated. The equivalent diameter of a drop is given by Eq. (6). The terminal velocity of the falling water drops was based on the data from Gunn and Kinzer [9].

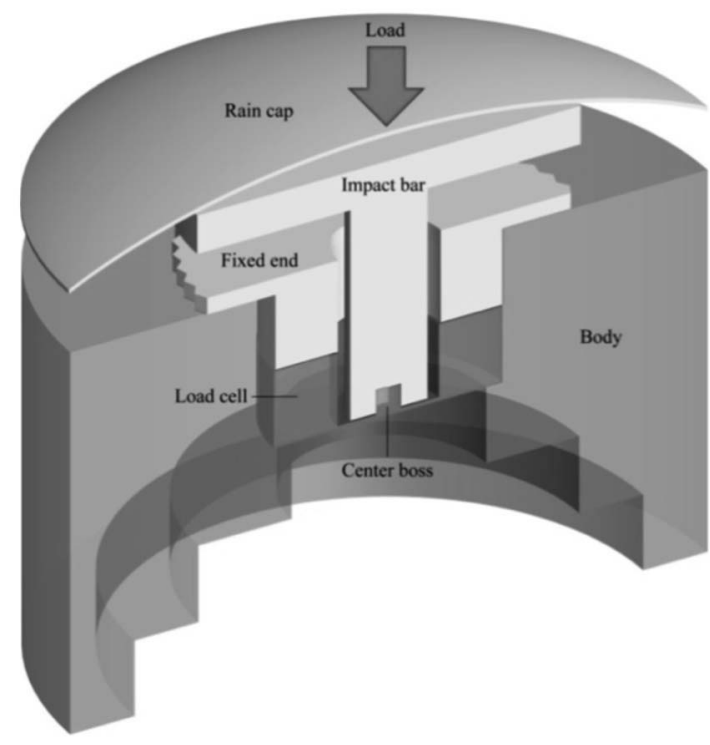

Fig. 3. Schematic view of the weighting distrometer.

Table 1. Characteristics of the water drops

\begin{tabular}{ccccc}
\hline Name & WD00 & WD01 & WD02 & WD03 \\
\hline \hline Mass of drop (g) & 0.0113 & 0.022 & 0.0565 & 0.0825 \\
\hline $\begin{array}{c}\text { Equivalent drop } \\
\text { Diameter (mm) } \\
\text { Terminal } \\
\text { velocity (m/sec) }\end{array}$ & 2.78 & 3.48 & 4.76 & 5.40 \\
\hline \hline
\end{tabular}

The weighting disdrometer measures the impact force of a falling raindrop and estimates the corresponding water drop in a static state.

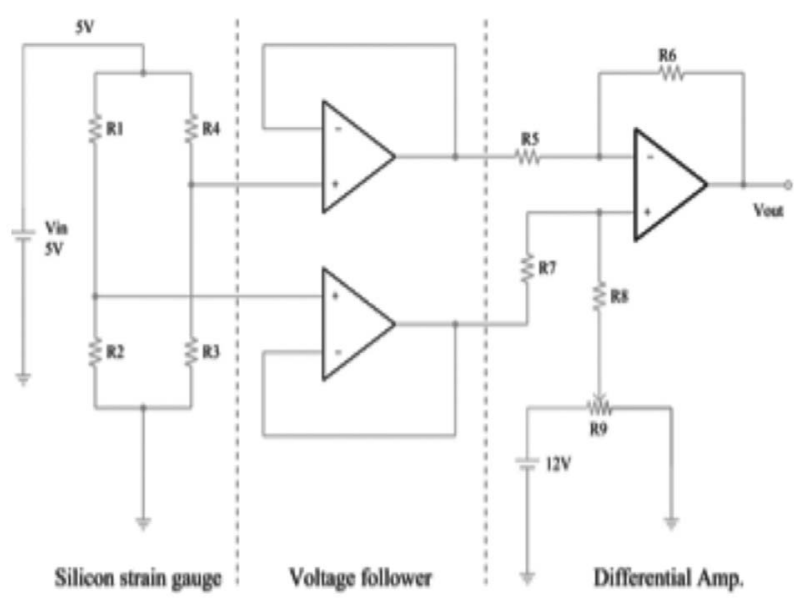

Fig. 4. Circuit diagram of the differential amplifier. 
The weighting disdrometer assume the size of the raindrop by measuring the impact force. The momentum of water drop $\left(M_{W D}\right)$ is given by its mass and velocity in Eq. (4).

$$
M_{W D}=m_{0} v_{0}
$$

Some momentum is lost by the surface state of the rain cap and the internal friction, because the raindrop is not an elastic body. The change in momentum of a raindrop is calculated using the force and time relationships of the raindrop [10]. The impulse $\left(I_{R C}\right)$ of the raindrop impacting a surface of the rain cap is expressed in Eq. (5),

$$
I_{R C}=\int_{t_{0}}^{t_{p}} F(t) d t=\bar{F} \Delta t=\Delta p
$$

where $\underline{F}$ is the average force of impact, $\Delta t$ is the impact duration time from $t_{0}$ to $t_{p}$, and $\Delta p$ is the change of momentum .

In general, naturally occurring raindrops are roughly spherical, and their size is from 0.1 to $8 \mathrm{~mm}$ in equilibrium diameter, which is approximated as the diameter of a complete sphere of the same volume [11]. More than $8 \mathrm{~mm}$ of an equivalent diameter raindrop is decomposed into small droplets. For a raindrop with mass $(m)$, the equilibrium diameter $(D)$ of the raindrop is given by Eq. (6),

$$
D=10 \times \sqrt[3]{\frac{6 m}{\rho \pi}}[\mathrm{mm}]
$$

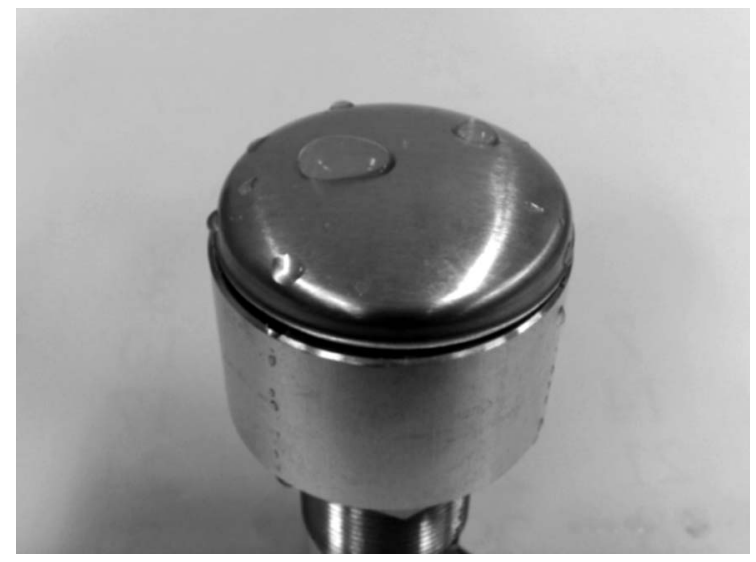

Fig. 5. The photo graph of the weighting disdrometer. where $m[\mathrm{~g}]$ is the mass of the raindrop and $\rho$ is the density of the raindrop. The measuring range of the weighting distrometer along the diameter of raindrops is from 1 to $6 \mathrm{~mm}$.

In addition, the amount of precipitation can be calculated from the value, which is divided by the area of the rain cap in the sum of the each raindrop volume.

Figure 5 shows the weighting disdrometer for measuring the dynamic load of raindrop.

\section{RESULT}

\subsection{Output characteristics of the load cell by static load}

Characteristics of the load cell were confirmed with a static load of coins, as shown in Fig. 6 (a).

The change in output voltages of the load cell with the differential amplifier due to changes in the vertical static load, sequentially reduced by intervals of $7.69 \mathrm{~g}$ from 76.9 to $0 \mathrm{~g}$, can be seen in Fig. 6 (b). When the thickness of the diaphragm is $250 \mu \mathrm{m}$, the sensitivity of the load cell in the static load $\left(\mathrm{S}_{\mathrm{L}} / 1000\right)$ is $7.8 \mathrm{mV} / \mathrm{g}$, which is controlled by the doping concentration of the piezoresistors and the thickness of the diaphragm as well as the amplification ratio of the circuit. If the mass constant of the load cell and the gauge factor of the strain gauge are $\mathrm{K} \cdot \mathrm{GF}=19.32$, the output voltage of the weighting disdrometer is almost the same as the value of Eq. (3), as show in Fig. 6 (c).

\subsection{Output characteristics of the disdrometer by impact load}

A weighting disdrometer with a diaphragm $250 \mu \mathrm{m}$ thick was used for measurement of observed impact loads of water drops falling into the center of the rain cap under wet conditions. Average values of the impulse for 4 drops, depending on the distance of the falling WD01, are show in Fig. 7. The peak voltage of the weighting disdrometer and the time duration will also vary depending on the status of the rain cap and wind velocity. In order to calibrate, there is a need to obtain the values of the output momentum, that is, peak voltage $\left(V_{p}\right)$ multiplied by the impact duration time $(\Delta t)$.

At more than $14 \mathrm{~m}$ in height, the output momentum of a water drop is saturated due to the decrease in acceleration 
of the falling water drop from air resistance.

The output signal characteristics of the weighting disdrometer from the collision of a water drop from a height

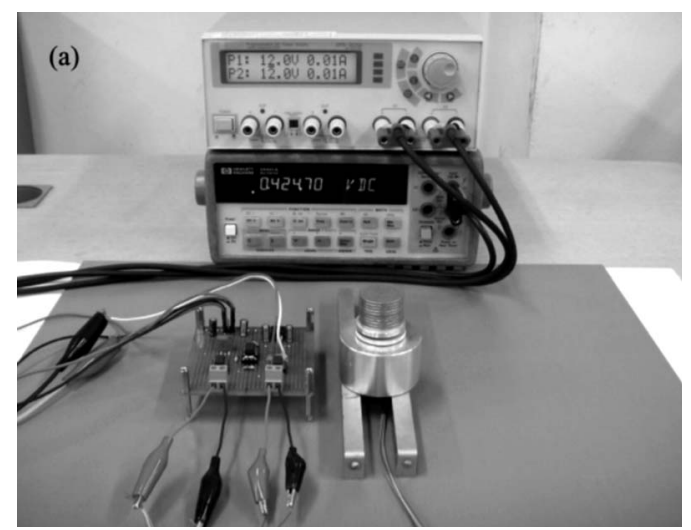

(a)

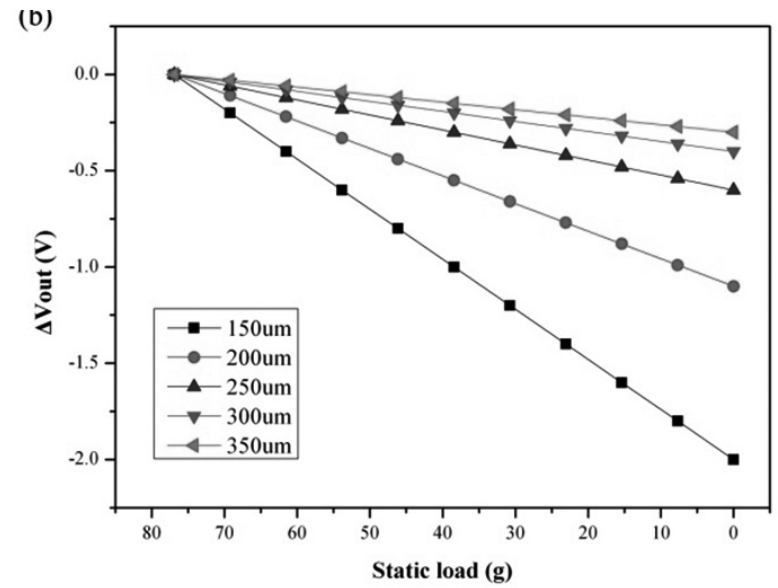

(b)

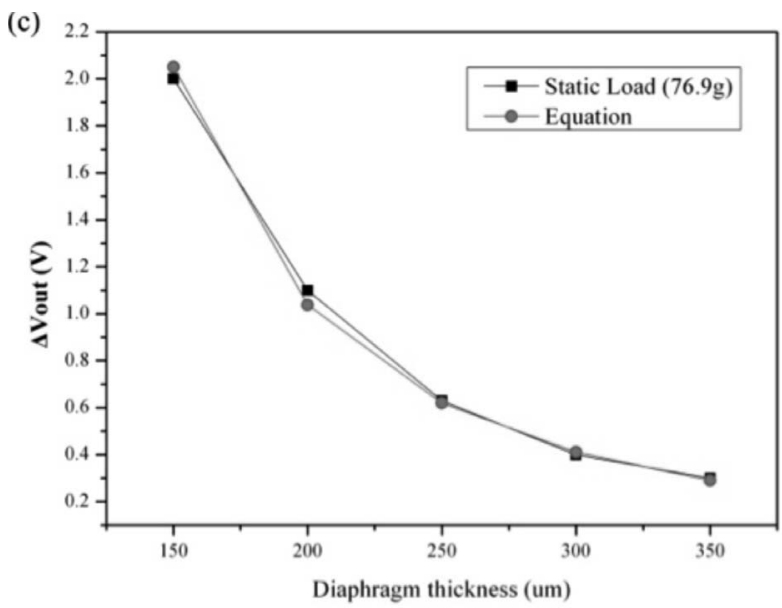

(c)

Fig. 6. Static load measurement; (a) Measurement setup, (b) output voltages of the load cell according to the static load, and (c) output voltages according to the thickness of the diaphragm. of $14 \mathrm{~m}$ are shown in Fig. 8. When the water drop hits the rain cap of the disdrometer, a part of momentum is transferred to the load cell, and the diaphragm vibrates until the stress wave ends. The average $V_{p}$ of WD01 falling from a height of $14 \mathrm{~m}$ was measured at $727.7 \mathrm{mV}$. The impact duration time $(\Delta t)$ is $0.1625 \mathrm{~ms}$ and the output momentum, the output voltage multiplied by $\Delta t$, is $0.1183 \mathrm{mVs}$.

The output momentum of the weighting distrometer represents good linearity according to the mass of droplets, as shown in Fig. 9. When considering the loss of momentum, from Eq. (4-5), the momentum of the water drop (MWD) and the output momentum of the weighting disdrometer (IRC) can be approximated by the relationship,

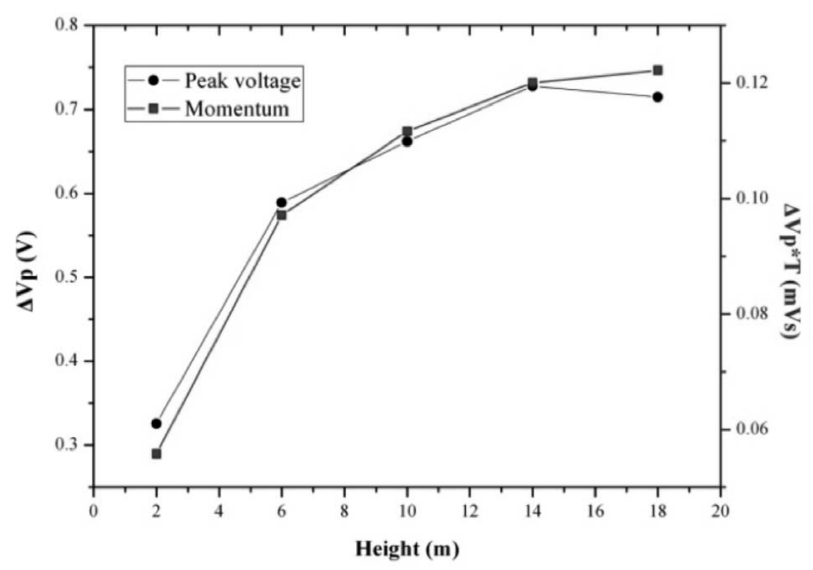

Fig. 7. The output characteristics of the weighting disdrometer according to the height of the water drop.

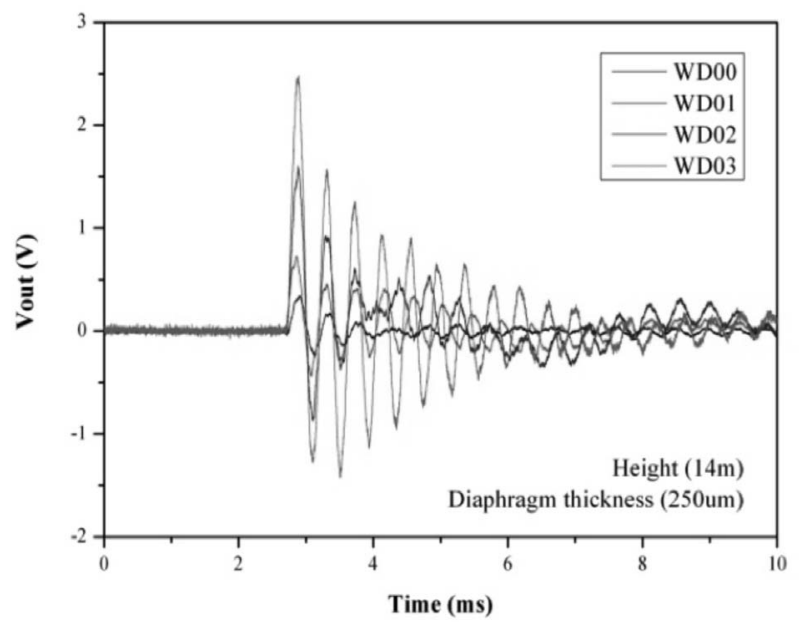

Fig. 8. Output signal of the weighting disdrometer during the impact of water drop. 


$$
\frac{K_{D}\left(I_{R C}\right)+c}{S_{I}}=M_{W D}
$$

where $K_{D}$ is the correction factor, c is the appropriate constant and $S_{L}$ is the sensitivity of the load cell in the static load.

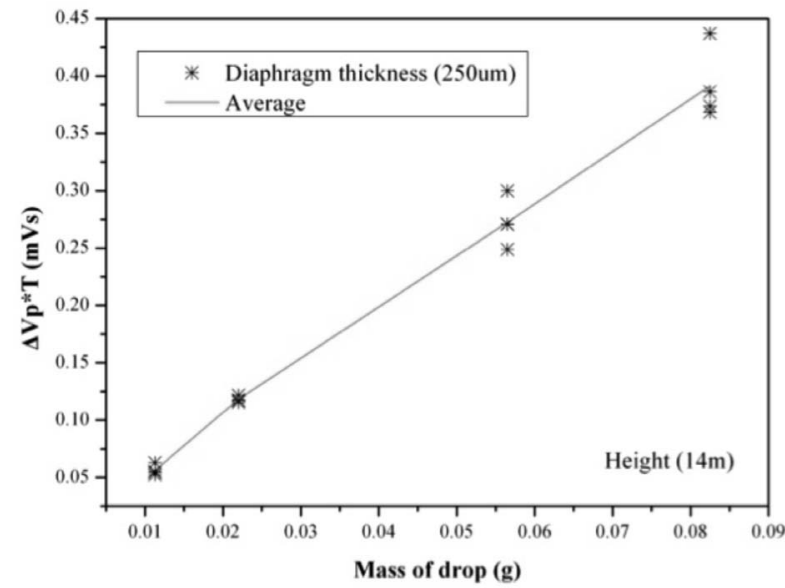

Fig. 9. The output momentum of the weighting distrometer.

\section{CONCLUSION}

A new type of weighting disdrometer with a silicon strain gauge load cell has been developed in order to measure the impact load of raindrops. The spring element of the silicon strain gauge load cell was made of the SUS630 for the center boss diaphragm, and the sensing element was fabricated by using a MEMS process for the silicon strain gauge. Therefore, the weighting disdrometer was able to provide good linear output and high sensitivity for the meteorological instrument. These properties demonstrated the capability of the silicon strain gauge load cell in a disdrometer.

\section{ACKNOWLEDGEMENTS}

This research was supported by the Small \& Medium Business Administration (SMBA) funded by the Korea government (S2045113) and the BK21 plus program in 2013.

\section{REFERENCES}

[1] R. Bagree, Characterization and design of a readout circuit for a piezoelectric-based acoustic disdrometer, Delft University of Technology, 2012.

[2] N. M. White and J. E. Brignell, "A planar thick film load cell”, Sens. Actuator A-Phys,. Vol. 25-27, pp. 313319, 1991.

[3] Y. Kim, S. Kwon, "Resistivity dependence of gauge factor of polysilicon strain gauge", Micro Nano Lett., Vol. 5, pp. 189-192, 2010.

[4] A. Yasukawa, M. Shimazoe, and Y. Matsuoka, "Simulation of circular silicon pressure sensors with a center boss for very low pressure measurement", IEEE Trans. Electron Devices, Vol. 36, No. 7, pp. $1295-$ 1302, 1989.

[5] Y. S Moon, S. G Lee, S. H. Ryu, and S. Y. Choi, "The silicon type load cell with SUS630 diaphragm", $J$. Sensor Sci. \& Tech., Vol. 20, No. 3, pp. 213-218, 2011.

[6] S. G. Lee, Y. S. Moon, W. H. Son, S. Y. Kwon, and S. Y. Choi, "The design considerations of the silicon strain gauges on a metal diaphragm for improving the sensitivity and accuracy of the pressure sensor", ICEIC pp. 664-665, 2013.

[7] Y. Kanda, "A graphical representation of the piezoresistance coefficient in silicon", IEEE Tran. Electron Devices, Vol. ED-29, No. 1, pp. 64-70, 1982.

[8] J. D. Dally and W. F. Riley, Experimental Stress Analysis, Edited by B. J. Clark, McGraw-Hill Kogakusha, Chapter 8, pp. 217-272, 1978.

[9] R. Gunn and G. D. Kinzer, "The terminal velocity of fall for waterdroplets in stagnant air", J. Meteor., Vol. 6, pp. 243-248, 1949.

[10] M. A. Nearing, J. M. Bradford, and R. D. Holtz, "Measurement of force vs. time relations for waterdrop impact", Soil Sci. Soc. Am. J., Vol. 50, No. 6, pp. 1532-1536, 1986.

[11] K. V. Beard and C. Chuang, "A new model for the equilibrium shape of raindrops", J. Atmos. Sci, Vol. 44, No. 11, pp. 1509-1524, 1987. 